\title{
Analysis of Phenolic Acids of Jerusalem Artichoke (Helianthus tuberosus L.) Responding to Salt-Stress by Liquid Chromatography/Tandem Mass Spectrometry
}

\author{
Fujia Chen, ${ }^{1}$ Xiaohua Long, ${ }^{1}$ Zhaopu Liu, ${ }^{1}$ Hongbo Shao, ${ }^{2,3}$ and Ling Liu ${ }^{1}$ \\ ${ }^{1}$ Key Laboratory of Marine Biology Jiangsu Province, College of Resources and Environmental Sciences, Nanjing Agricultural University, \\ Nanjing 210095, China \\ ${ }^{2}$ Key Laboratory of Coastal Biology \& Bioresources Utilization, Yantai Institute of Coastal Zone Research, \\ Chinese Academy of Sciences (CAS), Yantai 264003, China \\ ${ }^{3}$ Jiangsu Academy of Agricultural Sciences, Nanjing 210014, China
}

Correspondence should be addressed to Hongbo Shao; shaohongbochu@126.com and Ling Liu; liuling@njau.edu.cn

Received 24 May 2014; Accepted 6 July 2014; Published 5 August 2014

Academic Editor: Marian Brestic

Copyright (C) 2014 Fujia Chen et al. This is an open access article distributed under the Creative Commons Attribution License, which permits unrestricted use, distribution, and reproduction in any medium, provided the original work is properly cited.

Plant phenolics can have applications in pharmaceutical and other industries. To identify and quantify the phenolic compounds in Helianthus tuberosus leaves, qualitative analysis was performed by a reversed phase high-performance liquid chromatography coupled with tandem mass spectrometry (HPLC-MS/MS) and quantitative analysis by HPLC. Ten chlorogenic acids (CGAs) were identified (3-o-caffeoylquinic acid, two isomers of caffeoylquinic acid, caffeic acid, $p$-coumaroyl-quinic acid, feruloylquinic acid, 3,4-dicaffeoyquinic acid, 3,5-dicaffeoylquinic acid, 1,5-dicaffeoylquinic acid, and 4,5-dicaffeoylquinic acid) by comparing their retention times, UV-Vis absorption spectra, and MS/MS spectra with standards. In addition, four other phenolic compounds, including caffeoyl glucopyranose, isorhamnetin glucoside, kaempferol glucuronide, and kaempferol-3-o-glucoside, were tentatively identified in Helianthus tuberosus leaves for the first time. The 3-o-caffeoylquinic acid (7.752 mg/g DW), 4,5-dicaffeoylquinic acid $(5.633 \mathrm{mg} / \mathrm{g} \mathrm{DW})$, and 3,5-dicaffeoylquinic acid $(4.900 \mathrm{mg} / \mathrm{g} \mathrm{DW})$ were the major phenolic compounds in leaves of Helianthus tuberosus cultivar NanYu in maturity. The variations in phenolic concentrations and proportions in Helianthus tuberosus leaves were influenced by genotype and plant growth stage. Cultivar NanYu had the highest concentration of phenolic compounds, in particular 3-o-caffeoylquinic acid and 4,5-dicaffeoylquinic acid compared with the other genotypes (wild accession and QingYu). Considering various growth stages, the concentration of total phenolics in cultivar NanYu was higher at flowering stage $(5.270 \mathrm{mg} / \mathrm{g}$ DW) than at budding and tuber swelling stages. Cultivar NanYu of Helianthus tuberosus is a potential source of natural phenolics that may play an important role in the development of pharmaceuticals.

\section{Introduction}

Helianthus tuberosus L. (Jerusalem artichoke), Asteraceae family, is a perennial herb originating from eastern North America. It has been introduced and cultivated widely in the temperate areas for the edible tubers. H. tuberosus has tall stem, large leaves, bright yellow flowers resembling those of sunflowers, and fleshy potato-like tubers. As a source of inulin, the tubers have been used as a folk medicine for the treatment of diabetes and rheumatism with a variety of pharmacological activities, such as aperient, cholagogue, diuretic, spermatogenic, stomachic, and tonic [1]. Additionally, the leaves of $H$. tuberosus have been utilized as a folk medicine for the treatment of bone fracture, skin wounds, swelling, and pain $[2,3]$ with antipyretic, analgesic, anti-inflammatory, and antispasmodic effects [4-6]. Moreover, the stalks and leaves of this plant were also found to possess antioxidant, antimicrobial, antifungal, and anticancer activities $[1,6,7]$.

The effective compounds in $H$. tuberosus are coumarins, unsaturatedfatty acids, polyacetylenic derivatives, phenolic compounds, and sesquiterpenes [1]. Recent studies have shown that pharmacological characteristics of $H$. tuberosus were related to its phenolic compounds with antioxidant and radical-scavenging activity; the main phenolic acids in 
H. tuberosus leaves were chlorogenic acids [6]. Chlorogenic acids had inhibitory effects on carcinogenesis in the large intestine, liver, and tongue and protective effects against oxidative stress in vivo [8]. More broadly, phenolic acids are widely distributed in plants as the secondary metabolites [9]; some phenolic acids are allelochemicals used to control biological pests [10-12], plant pathogens [13], and weeds [14]. The involvement of phenolics with plant protection and communication makes phenolics pivotal molecules in the responses of plants to their ever-changing environment [15].

Previously, it was demonstrated that the leaves of $H$. tuberosus contained high concentration of phenolic compounds [5]. Phenolics were separated and identified (such as ferulic acids) from the tubers of $H$. tuberosus [16]. However, to date, reports on analysis and identification of phenolic compounds from the leaves of $H$. tuberosus are scarce and only a few phenolics, especially chlorogenic acid and isochlorogenic acids, have been identified and qualitatively analysed [6].

Reversed phase high-performance liquid chromatography coupled to tandem mass spectrometry (HPLC-MS/MS) has been extensively and successfully applied to the online structure elucidation of phenolic compounds in foodstuffs, having advantages of high sensitivity, speed, and low sample consumption [17-24]. In addition, liquid chromatography coupled to tandem mass spectrometry (LC-MS/MS) techniques are useful for elucidating the structures of the active compounds (e.g., nonvolatile phenolic compounds) and distinguishing compounds with identical molecular weights [23, 25].

The objectives of the present work were to identify the phenolic compounds in $H$. tuberosus leaves, using HPLCMS/MS technique, and to measure the concentration of main phenolics in $H$. tuberosus leaves of different cultivars at different sampling periods from budding stage to maturity (tuber swelling stage) using HPLC.

\section{Materials and Methods}

2.1. Chemicals and Materials. Gallic acid was obtained from Sinopharm Chemical Reagent Co., Ltd. (Shanghai, China); and 3-o-caffeoylquinic acid was obtained from Aladdin Reagent Co., Ltd. (Shanghai, China). Other standard samples were obtained from Yuanye Biological Technology Co., Ltd. (Shanghai, China). All other analytical grade chemicals were obtained from Shoude Experimental Equipment Co., Ltd. (Nanjing, China).

The leaves of three $H$. tuberosus cultivars (the wild accession, the southern cultivar NanYu [26], and QingYu originated from northern China) were collected from Dafeng District (Jiangsu, China) in maturity at the end of October 2011. Both cultivars NanYu and QingYu [27, 28] are superior varieties in local areas which have obvious competitive advantages in yield, quality, saline-alkali tolerance, and so on over the wild accession in Dafeng District. The leaves of cultivar NanYu were collected from August to October 2012 at different growth stages including budding, flowering, and tuber swelling stages, respectively.
2.2. Extraction of Phenolic Compounds. The air-dried (room temperature) and milled [6] leaves ( $10 \mathrm{~g}$ ) of $H$. tuberosus were refluxed under vacuum at $50^{\circ} \mathrm{C}$ using $70 \%$ v/v ethanol $(\mathrm{EtOH})$ for three hours. After evaporation under reduced pressure, the dry residue was redissolved in $25 \mathrm{~mL}$ of methanol and used for colorimetric and chromatographic analyses. For HPLC analysis, all samples were filtered through a $0.22 \mu \mathrm{m}$ cellulose acetate filter (Millipore Corp., Bedford, MA, USA) before injections.

2.3. Measurement of Total Phenolic Concentration. The total phenolic concentration (TPC) was determined using the Folin-Ciocalteu reagent with gallic acid as standard [19, 29, 30]. The reaction mixture contained $0.5 \mathrm{~mL}$ of test sample, $0.5 \mathrm{~mL}$ of the Folin-Ciocalteu reagent freshly prepared in our laboratory, $2.0 \mathrm{~mL}$ of $10 \% \mathrm{w} / \mathrm{v}$ sodium carbonate solution, and $3.0 \mathrm{~mL}$ of distilled water. After $2 \mathrm{~h}$ of reaction under ambient temperature in the dark, the absorbance at $760 \mathrm{~nm}$ was measured. A calibration curve with equation: $y=$ $0.0029 x+0.0107\left(R^{2}=0.9991\right)$ was constructed using gallic acid solutions in the range of $1.470-294 \mathrm{mg} / \mathrm{L}$. Results were expressed in milligram gallic acid equivalents per gram of dried sample.

2.4. HPLC-MS/MS Analysis. HPLC-MS/MS analysis of phenolics in $H$. tuberosus extracts was performed using an Agilent 1200 series HPLC system (Agilent Technology Co. Ltd., USA), composed of a diode array detector and an Agilent 6400 series triple quadrupole (QQQ) mass spectrometer equipped with an electrospray ionization (ESI) source. Data were collected and processed via a personal computer running Agilent MassHunter workstation (Micromass, Qualitative Analysis Version B.01.03 of Agilent Technology Co. Ltd., USA). A reverse-phase Eclipse XDB-C18 column (250 mm $\times$ $4.6 \mathrm{~mm}, 5 \mu \mathrm{m}$, Agilent Technology Co. Ltd., USA) was used for separation. The mobile phases consisted of methanol (A) and $1.0 \% \mathrm{v} / \mathrm{v}$ formic acid aqueous solution (B). Gradient elution was started with $30 \%$ of $\mathrm{A}$ and ascended to $50 \%$ of $\mathrm{A}$ in $45 \mathrm{~min}$. The flow rate was kept at $0.8 \mathrm{~mL} / \mathrm{min}$, and the column temperature was $30^{\circ} \mathrm{C}$. Samples were filtered through a $0.22 \mu \mathrm{m}$ filter prior to HPLC injection. The injection volume was $5 \mu \mathrm{L}$. UV-Vis absorption spectra were recorded online from 200 to $600 \mathrm{~nm}$ during HPLC analysis. Phenolics were detected at the wavelength of $330 \mathrm{~nm}$.

Mass and MS/MS spectra were achieved by electrospray ionization (ESI) in negative modes. The voltages used were $4000 \mathrm{~V}$ for the source capillary and $10 \mathrm{~V}$ for the extraction cone: the source temperature was $150^{\circ} \mathrm{C}$ and the desolvation temperature was $350^{\circ} \mathrm{C}$. The electrospray probe flow was adjusted to $70 \mathrm{~mL} / \mathrm{min}$. The ESI-MS and ESI-MS/MS spectra were obtained by scanning from 200 to $1200 \mathrm{~m} / z$. The MS/MS fragmentations were carried out with 10\%-50\% energy.

2.5. Identification and Quantification of Phenolic Compounds. The phenolic compounds in $H$. tuberosus leaves extracts were identified by comparing their UV-Vis absorption spectra, matching their molecular ions $(\mathrm{m} / z)$ obtained by ESI-MS and ESI-MS/MS chromatographic characteristics with the 
TABLE 1: Identification of phenolic compounds in H. tuberosus leaves by HPLC-MS/MS.

\begin{tabular}{lcccccl}
\hline Peaks number & $t_{R}(\min )$ & $\mathrm{UV} \lambda_{\max }(\mathrm{nm})$ & $\mathrm{MW}$ & $\mathrm{MS}^{-}$ & $\mathrm{MS} / \mathrm{MS}$ & Identification \\
\hline 1 & 3.58 & $246.0,263.0$ & 360 & 359.4 & $297.3,281.6,230.9,135.2$ & Unknown \\
2 & 4.36 & $214.3,323.4$ & 354 & 353.4 & $191.1,179.1,161.1,135.1,85.1$ & Caffeoylquinic acid (isomer of chlorogenic acid) \\
3 & 5.78 & $214.4,327.0$ & 354 & 353.4 & $191.1,127.0,85.0$ & 3-o-Caffeoylquinic acid (3-CQA) \\
4 & 6.07 & $237.9,324.4$ & 354 & 353.4 & $191.2,127,93.1,85.0$ & Caffeoylquinic acid \\
5 & 8.57 & 323.0 & 180 & 179.1 & $136.0,107.9$ & Caffeic acid (CA) \\
6 & 9.19 & $239.1,311.5$ & 338 & 337.3 & $191.1,173.0,93.0$ & p-Coumaroyl-quinic acid \\
7 & 10.12 & $241.4,325.8$ & 368 & 367.3 & $191.0,173.1,134.0,93.0$ & Feruloylquinic acid \\
8 & 18.09 & $243.8,327.0$ & 516 & 515.5 & $354.3,191.1,173.1,179.1,135.0$ & Dicaffeoylquinic acid (3,4-DiCQA) \\
9 & 19.03 & $242.6,327.0$ & 516 & 515.5 & $354.1,191.2$ & Dicaffeoylquinic acid (3,5-DiCQA) \\
10 & 20.04 & 327 & 342 & 341.3 & $179.1,161.1$ & Caffeoyl glucopyranose \\
11 & 20.43 & $243,329.4$ & 516 & 515.5 & $354.1,191.1,179.1,173.1,135.1$ & Dicaffeoylquinic acid (1,5-DiCQA) \\
12 & 22.97 & $253.3,349.7$ & 478 & 477.4 & $315.3,300.1,270.9,180.2$ & Isorhamnetin glucoside \\
13 & 26.57 & 327.0 & 516 & 515.5 & $191.1,179.1,173.1,135.0$ & Dicaffeoylquinic acid (4,5-DiCQA) \\
14 & 30.20 & $263.9,341.3$ & 462 & 461.4 & $315.2,284.8,161.0,132.7,85.1$ & Kaempferol glucuronide \\
15 & 30.58 & $263.0,333.0$ & 448 & 447.4 & $285.4,190.8,153.1,96.9$ & Kaempferol-3-o-glucoside \\
\hline
\end{tabular}

literature data reported $[5,6]$ or with available reference standards. The external standard method was used for the quantification of main phenolic acids. Concentrations of 3-o-caffeoylquinic acid, caffeic acid, 3,4-dicaffeoylquinic acid, 3,5-dicaffeoylquinic acid, 1,5-dicaffeoylquinic acid, 4,5dicaffeoylquinic acid, and so on were calculated with the regression equations from the standard curves. Concentrations were expressed as $\mathrm{mg} / \mathrm{g}$ dried weight sample (DW).

2.6. Statistical Analysis. The TPC and concentration of phenolic compounds in $H$. tuberosus leaves of different cultivars and different growth stages were sources of variation. These data were reported as mean \pm SD from triplicate determinations. Statistical analysis was performed with analysis of variance (ANOVA) and statistical significance specified at $P \leq 0.05$.

\section{Results}

3.1. Identification of the Chromatographic Peaks. The examination of the chromatograms in a full-scan mode revealed the presence of several compounds, which were positively identified by comparison with available standards. Figure 1 showed the HPLC chromatogram of ethanol extract from $H$. tuberosus leaves. There were 15 phenolic peaks separated in extracts using the reversed phase C-18 column. As shown in Table 1, peak identification was performed by comparing retention times $\left(t_{R}\right)$, UV-Vis spectra, mass, and MS/MS spectra with those of reference standards or literature data.

Classically, chlorogenic acids (CGAs) are a family of esters formed between quinic acid and certain transcinnamic acids, most commonly caffeic, $p$-coumaric, and ferulic acids [31]. Fragment ions $m / z 191$ and $m / z$ 179, corresponding to deprotonated quinic acid and caffeic acid fragments, were characteristics of the MS/MS spectra of quinic or caffeic acid derivatives [32].

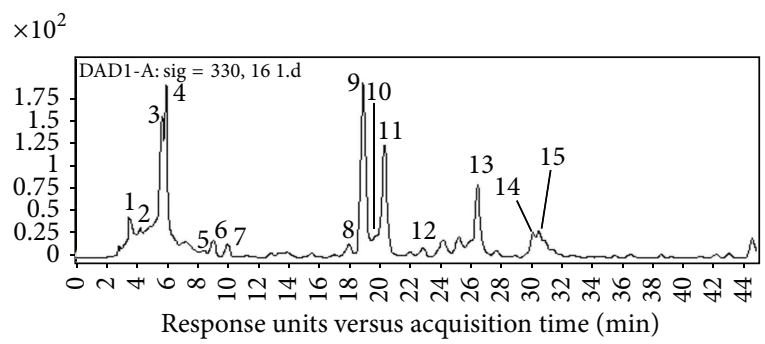

FIGURE 1: HPLC chromatogram of the ethanol extract of $H$. tuberosus leaves detected at $330 \mathrm{~nm}$. Peak numbers were consistent with those shown in Table 1.

Among all the peaks in the chromatogram (Figure 1), peak 9 was quite prominent, indicating a predominant phenolic compound in $H$. tuberosus leaves. This peak presented spectral characteristics of the dicaffeoylquinic acid $[5,6]$ with $\mathrm{UV} \lambda_{\max }$ at 242.6 and $327.0 \mathrm{~nm}$ and $t_{R}$ of $19.03 \mathrm{~min}$. The ESI-MS/MS spectra showed $[\mathrm{M}-\mathrm{H}]^{-}$at $m / z 515.5$, fragment ion $\left[\mathrm{M}-\mathrm{C}_{9} \mathrm{H}_{6} \mathrm{O}_{3}\right]^{-}$at $\mathrm{m} / z$ 354.1, and fragment ion [M$\left.\mathrm{H}-2 \mathrm{C}_{9} \mathrm{H}_{6} \mathrm{O}_{3}\right]^{-}$at $m / z 191.2$ (Figures 2(c), 2(d), 2(e), and $2(\mathrm{f}))$. Compared with the standard, this compound was unambiguously identified as 3,5-dicaffeoylquinic acid. Peaks 8,11 , and 13 had the same spectral characteristics as peak 9 (Table 1), with UV $\lambda_{\max }$ at 243.8 and $327.0 \mathrm{~nm}$ (peak 8), 243.0 and $329.4 \mathrm{~nm}$ (peak 11), and $327.0 \mathrm{~nm}$ (peak 13). Based on the MS/MS analyses, the caffeoylquinic acid $\mathrm{m} / z 354.1$ ion further fragmented to form characteristic $\mathrm{m} / z$ 173.1 [M$\left.\mathrm{H}-2 \mathrm{C}_{9} \mathrm{H}_{6} \mathrm{O}_{3}-\mathrm{H}_{2} \mathrm{O}\right]^{-}, 135.0$ [M- $\left.\mathrm{C}_{7} \mathrm{H}_{10} \mathrm{O}_{5}-\mathrm{C}_{9} \mathrm{H}_{6} \mathrm{O}_{3}-\mathrm{COOH}\right]^{-}$, and $179.0\left[\mathrm{M}-\mathrm{H}-\mathrm{C}_{9} \mathrm{H}_{6} \mathrm{O}_{3}-\mathrm{C}_{7} \mathrm{H}_{10} \mathrm{O}_{5}\right]^{-}$ions [33]. Compared with the standard, these compounds were identified as 3,4-dicaffeoylquinic acid, 1,5-dicaffeoylquinic acid, and 4,5dicaffeoylquinic acid, respectively [34].

The peaks 2,3, and 4 were identified as three isomers of caffeoylquinic acids (chlorogenic acid) based on the detailed fragmentation, UV absorption, and also [35]. Previously, 


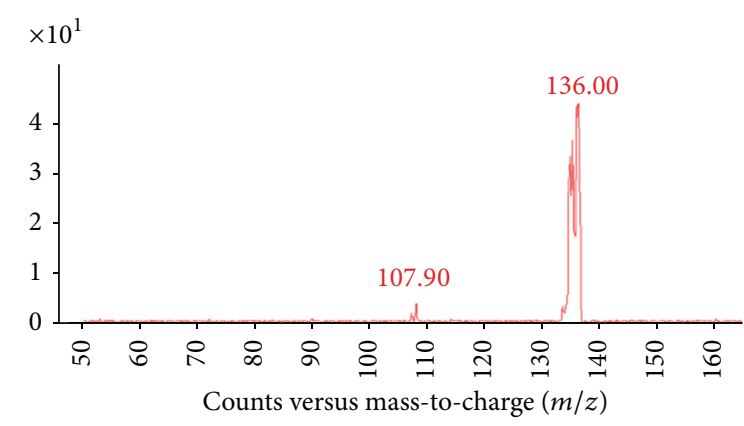

(a)

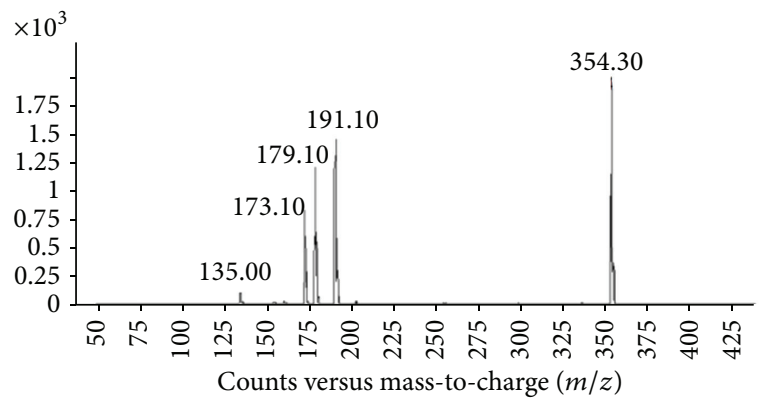

(c)

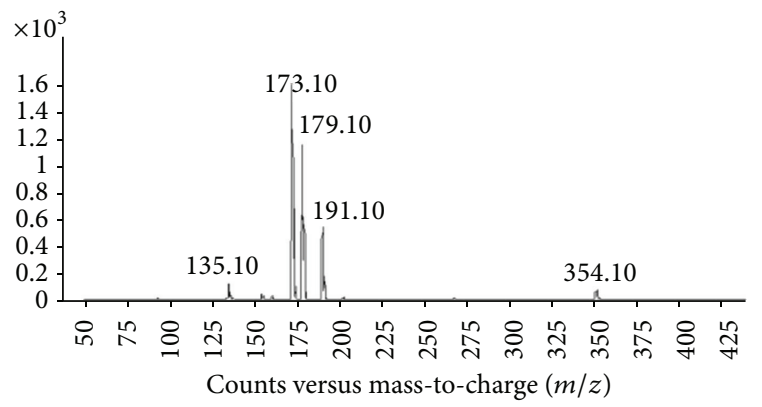

(e)

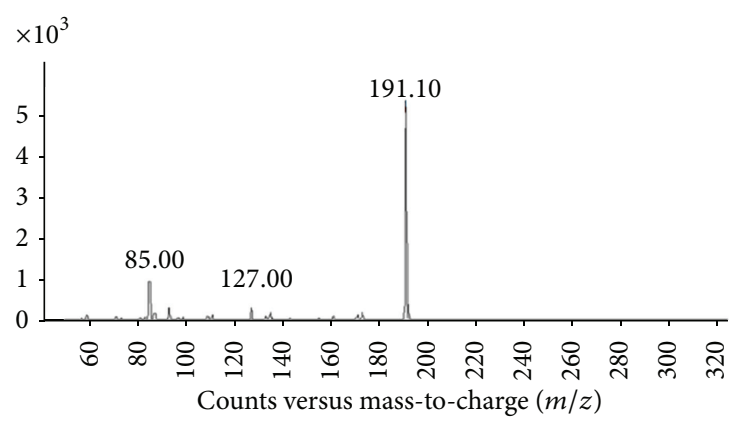

(b)

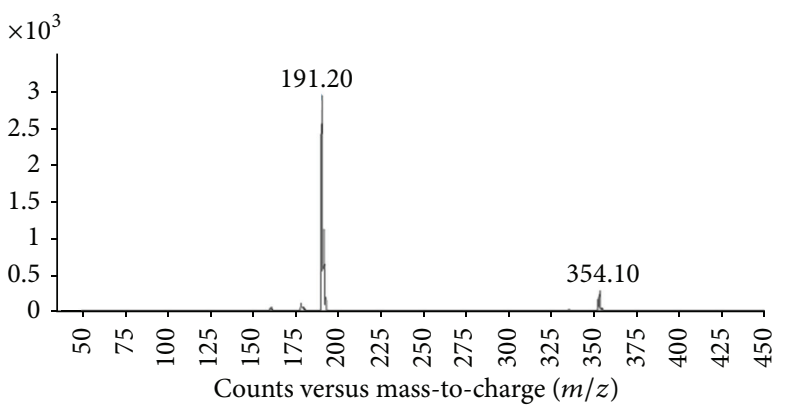

(d)

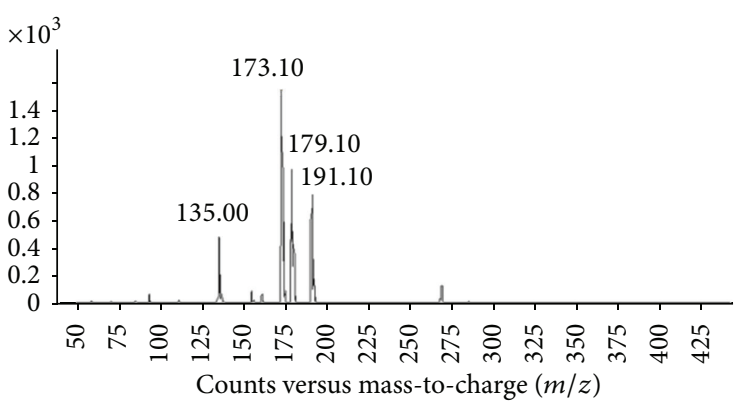

(f)

FIGURE 2: HPLC-MS-MS spectra of phenolic acids ( $m / z 179$ of caffeic acid (a), $m / z 353$ of 3-o-caffeoylquinic acid (b), $m / z 515$ of 3,4dicaffeoylquinic acid (c), $m / z 515$ of 3,5-dicaffeoylquinic acid (d), $m / z 515$ of 1,5-dicaffeoylquinic acid (e), and $m / z$ 515 of 4,5-dicaffeoylquinic acid (f)).

the presence of cis derivatives of chlorogenic acids was reported in coffee leaves, Rudbeckia hirta, Carlina acaulis, H. tuberosus, Symphyotrichum novae-angliae, maté tea (Ilex paraguariensis), and leaves of other Asteraceae plants [31,3638]. In a negative ion ESI mode, the deprotonated molecule $[\mathrm{M}-\mathrm{H}]^{-}$at $m / z 353$ and fragment ion [M-H-C $\left.\mathrm{C}_{6} \mathrm{H}_{3}\right]^{-}$ formed from deprotonated quinic acid at $m / z 191$ were observed (Figure 2(b)). Fragment ions $m / z 85$ and $m / z$ 93, characteristic of the quinic acid moiety of monoacyl and diacyl chlorogenic acids, defined the parent ions of putative chlorogenic acids [36]. Other fragment ions with different energies such as the caffeic acid unit $(\mathrm{m} / z$ 179.1) and [M$\left.\mathrm{H}-\mathrm{C}_{7} \mathrm{H}_{12} \mathrm{O}_{6}\right]^{-}(m / z$ 161.1) were used to distinguish the three isomers [38]. Compared with the standard, peak 3 was identified as 3-o-caffeoylquinic acid.

The peak 5 was pseudomolecular ion $[\mathrm{M}-\mathrm{H}]^{-}$at $\mathrm{m} / z$ 179.1 and fragment ions at $m / z 136.0$ [M-COO] $^{-}$and 107.9 [M-COO-CO $^{-}$(Figure 2(a)), which were the typical masses of caffeic acid in the negative mode [39]. Fragment ions at $\mathrm{m} / z 191$ and 179 were also observed in ESI-MS/MS ${ }^{-}$spectra of peaks 6,7 , and 10 (Table 1 ) indicating that they were derivatives of quinic acid or caffeic acid. Peak 6 was eluted at $9.19 \mathrm{~min}$ (Figure 1), with the molecular ion at $\mathrm{m} / z 337.3$ [M$\mathrm{H}]^{-}$and the main fragment ions at $m / z 191.1$ [quinic acid- $\left.\mathrm{H}\right]^{-}$

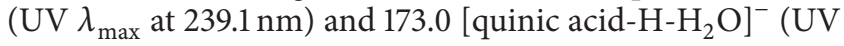
$\lambda_{\text {max }}$ at $311.5 \mathrm{~nm}$ ); this peak was identified as $p$-coumaroylquinic acid [31, 36, 40]. Peak 7 (Table 1) was identified as feruloylquinic acid ([M-H] $]^{-}$at $m / z 367$ and $U V \lambda_{\max }$ at 241.4 and $325.8 \mathrm{~nm})[5,32]$.

The MS/MS spectrum of peak 10 (Table 1) suggested caffeoyl glucopyranose that possesses both molecular ion $\mathrm{m} / z 341.3$ and fragmental ion $\mathrm{m} / z 179.1$ formed by loss of one dehydrated molecule of glucose (Glc) [M-H-(Glc$\left.\left.\mathrm{H}_{2} \mathrm{O}\right)\right]^{-}, 161.1\left[\left(\mathrm{Glc}-\mathrm{H}_{2} \mathrm{O}\right)-\mathrm{H}\right]^{-}[33,41]$. To our knowledge, caffeoyl glucopyranose has not been previously reported in H. tuberosus leaves. 
TABLE 2: Concentration of total phenolics and phenolic compounds in $H$. tuberosus leaves (cv. NanYu).

\begin{tabular}{lc}
\hline Phenolic compounds $^{\mathrm{b}}$ & Concentration $^{\mathrm{a}}(\mathrm{mg} / \mathrm{g}$ dry weight) \\
\hline Caffeoylquinic acid (peak 2) $^{\mathrm{b}}$ & $0.063 \pm 0.008^{\mathrm{d}}$ \\
3-o-Caffeoylquinic acid & $7.752 \pm 2.872^{\mathrm{b}}$ \\
Caffeoylquinic acid (peak 4) $^{\mathrm{b}}$ & $0.538 \pm 0.081^{\mathrm{d}}$ \\
Caffeic acid & $0.098 \pm 0.052^{\mathrm{d}}$ \\
p-Coumaroyl-quinic acid $^{\mathrm{d}}$ & $0.153 \pm 0.061^{\mathrm{d}}$ \\
Feruloylquinic acid & $0.527 \pm 0.199^{\mathrm{d}}$ \\
3,4-Dicaffeoylquinic acid & $0.618 \pm 0.215^{\mathrm{d}}$ \\
3,5-Dicaffeoylquinic acid & $4.900 \pm 1.492^{\mathrm{c}}$ \\
Caffeoyl glucopyranose & $0.001 \pm 0.319^{\mathrm{d}}$ \\
1,5-Dicaffeoylquinic acid & $1.733 \pm 0.567^{\mathrm{d}}$ \\
Isorhamnetin glucoside $^{\mathrm{d}}$ & $0.348 \pm 0.057^{\mathrm{d}}$ \\
4,5-Dicaffeoylquinic acid & $5.633 \pm 2.990^{\mathrm{bc}}$ \\
Kaempferol glucuronide $^{\mathrm{d}}$ & $0.186 \pm 0.034^{\mathrm{d}}$ \\
Kaempferol-3-o-glucoside $^{\mathrm{d}}$ & $1.020 \pm 0.379^{\mathrm{d}}$ \\
Total phenolics $^{\mathrm{e}}$ & 23.570 \\
Total phenolics $^{\mathrm{f}}$ & $30.159 \pm 4.410^{\mathrm{a}}$ \\
\hline
\end{tabular}

${ }^{\mathrm{a}}$ Values are expressed as mean \pm SD of triplicate measurements; the means in a column followed by the same letters represent values that are not significantly different according to Duncan's test $(P \leq 0.05)$; ${ }^{\mathrm{b}}$ quantified as 3-o-caffeoylquinic acid; ${ }^{c}$ quantified as caffeic acid; ${ }^{\mathrm{d}}$ quantified as glucoside; ${ }^{\mathrm{e}}$ sum of the individual phenolic compounds; and ${ }^{\mathrm{f}}$ quantified as gallic acid equivalents.

The MS/MS analysis of peaks 12 , 14, and 15 (Table 1 ) showed fragment ions at $m / z 315,301$, and 285 , corresponding to methyl quercetin or methoxy kaempferol, quercetin aglycone, and kaempferol, suggesting that they were kaempferol and quercetin glycoside derivatives [33]. Peak 12 had a molecular ion $[\mathrm{M}-\mathrm{H}]^{-}$at $\mathrm{m} / z 477$ and fragment ions at $\mathrm{m} / z 315$ [M-H- $\left.\left(\mathrm{Glc}-\mathrm{H}_{2} \mathrm{O}\right)\right]^{-}, 300.1$ [M-H- $\left.\left(\mathrm{Glc}-\mathrm{H}_{2} \mathrm{O}\right)-\mathrm{CH}_{3}\right]^{-}$, and $270.9\left[\mathrm{M}-\mathrm{H}-\left(\mathrm{Glc}-\mathrm{H}_{2} \mathrm{O}\right)-\mathrm{CH}_{3}-\mathrm{CO}\right]^{-}$, which proved to be isorhamnetin glucoside [23,33]. Peaks 14 and 15 were possibly kaempferol glucuronide and kaempferol-3-o-glucoside, which have similar fragment ion 285 [kaempferol- $\mathrm{H}]^{-}$and different parent ions $461[\mathrm{M}-\mathrm{H}]^{-}$and $447[\mathrm{M}-\mathrm{H}]^{-}[23$, $32,33,42-44]$. These kaempferol and quercetin glycoside derivatives (peaks 12, 14, and 15) are also the first ever reports in Helianthus tuberosus leaves. Their exact structures need further confirmation and additional NMR data will be required.

Phenolics in peak 1 (Table 1) in the HPLC chromatogram were not identified.

3.2. Quantification of Phenolics. Concentration of phenolic compounds in $\mathrm{H}$. tuberosus leaves of cultivar $\mathrm{NanYu}$ was determined by the HPLC method, whereas the concentration of total phenolics was calculated as the sum of the individual phenolic compounds and was also estimated by using the Folin-Ciocalteu method (Table 2). The 3-ocaffeoylquinic acid $(7.752 \mathrm{mg} / \mathrm{g} \mathrm{DW})$, 4,5-dicaffeoylquinic acid $(5.633 \mathrm{mg} / \mathrm{g} \mathrm{DW})$, and 3,5-dicaffeoylquinic acid $(4.900 \mathrm{mg} / \mathrm{g} \mathrm{DW})$ were the major phenolic compounds in $H$. tuberosus leaves, and their concentrations accounted for $33 \%$,
$24 \%$, and $21 \%$ of the total phenolics, respectively. Among all the quantified phenolics, chlorogenic acids (CGAs) including 3-o-caffeoylquinic acid, caffeoylquinic acids (peaks 2 and 4), caffeic acid, $p$-coumaroyl-quinic acid, feruloylquinic acid, 3,4-dicaffeoylquinic acid, 3,5-dicaffeoylquinic acid, 1,5dicaffeoylquinic acid, and 4,5-dicaffeoylquinic acid contributed to the total of $22.015 \mathrm{mg} / \mathrm{g} \mathrm{DW}$ ( $93 \%$ of the total phenolics).

As shown in Table 2, the content of total phenolics calculated as the sum of the individual phenolic compounds was $23.570 \mathrm{mg} / \mathrm{g} \mathrm{DW}$, whereas the value obtained by the Folin-Ciocalteu method was $30.159 \mathrm{mg} / \mathrm{g}$ DW. The substantial difference between the two values was likely due to the interference of other reducing substances in phenolic extracts, leading to overestimation of total phenolic contents in the Folin-Ciocalteu colorimetric analysis $[45,46]$.

Concentration of six main phenolic compounds (peaks $3,5,8,9,11$, and 13 in Table 1 ) in leaves of different $H$. tuberosus cultivars sampled at different periods from budding to tuber swelling stages was presented in Figure 3. Among the tested genotypes of H. tuberosus (Figure 3(a)), NanYu had the highest concentration of phenolic compounds in leaves (around 7-fold higher than the wild accession and 3-fold higher than QingYu).

Caffeic acid was detected in low concentration in all genotypes, whereas concentrations of 3-o-caffeoylquinic acid and 4,5-dicaffeoylquinic acid were considerably higher in all cultivars investigated (Figure 3(a)).

Concentration of total phenolics in leaves of cultivar NanYu was higher at flowering stage $(5.270 \mathrm{mg} / \mathrm{g} \mathrm{DW})$ than budding and tuber swelling stages (Figure 3(b)), frombudding, flowering to tuber swelling stages.

\section{Discussion}

Phenolic acids are secondary metabolites that are commonly found in plant-derived foods. They have attracted considerable interest due to their many potential health benefits, which are powerful antioxidants and have been reported to demonstrate antibacterial, antiviral, anticarcinogenic, antiinflammatory, and vasodilatory actions [47]. As allelochemicals, the phenolic acids might play an important role in plant defense against pathogens [48], pests, and weeds [14, 49]. The mechanism of a phenolic with defense, communication, and protection roles was considered as a pivotal molecule in the responses of plants to their ever-changing environment [15].

The variation in concentration of phenolic acids reported here and in the literature was probably due to the isomerisation of chlorogenic acids (CGAs) [50] and different $H$. tuberosus parts considered (tubers, leaves, or whole plants) $[6,9,31]$. In addition, the phenolic profiles of Helianthus tuberosus leaves of cultivar NanYu (Dafeng District, Jiangsu, China) were different from previous studies in which the major phenolic compounds were 3-o-caffeoylquinic acid and 1,5-dicaffeoylquinic acid [6] in H. tuberosus leaves from Yulin District (Shannxi, China), probably due to different cultivars of $H$. tuberosus, different sampling periods, or different origins. 


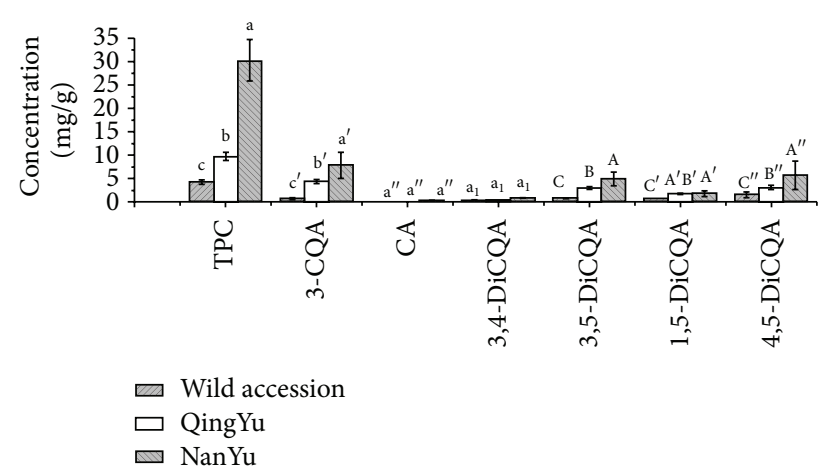

(a)

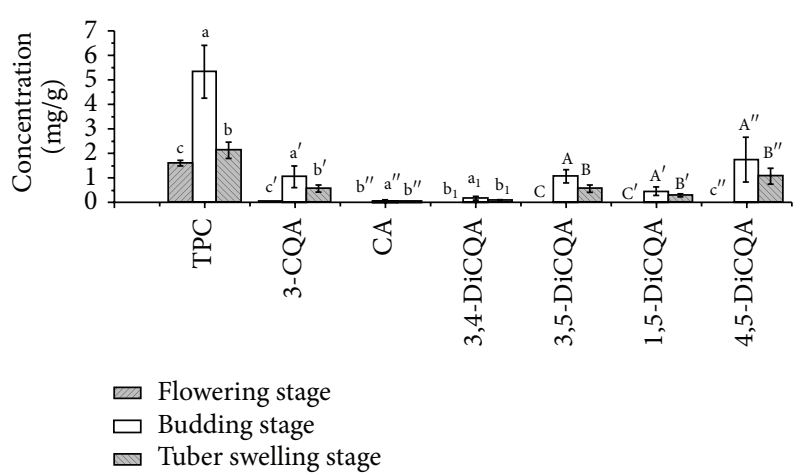

(b)

FIGURE 3: Concentration of phenolics in H. tuberosus leaves of different genotypes: (a) in 2011 and growth stages of cultivar NanYu (flowering stage, budding, and tuber swelling stages) and (b) from August to October in 2012. Concentrations were in mg/g dry weight of leaves. Values are expressed as mean \pm SD of triplicate measurements; columns with the same letters are not significantly different according to Duncan's test $(P \leq 0.05)$.

However, this was to be expected as there were so many environmental factors such as pedoclimatic (soil type, sun exposure, and rainfall) and agronomic factors (growth in greenhouses or fields, biological culture, hydroponic culture, fruit yield per tree, etc.) that could affect phenolics concentration in plants [51]. A degree of ripeness also considerably affected the concentrations and proportions of various phenolics [52]. Thus, cultivar NaYu can be a potential source of natural phenolics, which could have multiple functions (e.g., pharmaceuticals) and could play an important role in plant interactions and ecosystem patterning [14].

\section{Conclusions}

Reversed phase high-performance liquid chromatography coupled with tandem mass spectrometry (HPLC-MS/MS) was successfully employed in the qualitative analysis of phenolic compounds in $H$. tuberosus leaves. Ten chlorogenic acids (CGAs) were identified (3-o-caffeoylquinic acid, two isomers of caffeoylquinic acids, caffeic acid, $p$-coumaroylquinic acid, feruloylquinic acid, 3,4-dicaffeoylquinic acid, 3,5-dicaffeoylquinic acid, 1,5-dicaffeoylquinic acid, and 4,5dicaffeoylquinic acid), and four others (caffeoyl glucopyranose, isorhamnetin glucoside, methoxy kaempferol glucoside, and kaempferol-3-o-glucoside) were tentatively identified for the first time. Quantitative analysis of phenolics indicated that 3-o-caffeoylquinic acid, 4,5-dicaffeoylquinic acid, and 3,5-dicaffeoylquinic acid were the three major phenolic compounds in $H$. tuberosus leaves. The variation in phenolic concentrations and proportions in $H$. tuberosus leaves was characterised in different genotypes and at different sampling periods from budding to tuber swelling stages. $H$. tuberosus cultivar $\mathrm{NaYu}$ had the highest concentration of total phenolics and might be a potential source of natural phenolics, which could play an important role in the development of pharmaceuticals.

\section{Conflict of Interests}

The authors declare that there is no conflict of interests regarding the publishing of this paper.

\section{Authors' Contribution}

Fujia Chen and Xiaohua Long contributed to the work of the paper equally.

\section{Acknowledgments}

The authors are grateful for the financial support of Jiangsu Agricultural Science and Technology Independent Innovation Fund Project (no. CX(12)1005-6), National Natural Science Foundation of China (no. 31201692; 41171216), the National Key Projects of Scientific and Technical Support Programs funded by the Ministry of Science and Technology of China (no. 2011BAD13B09), the Ministry of Science and Technology of Jiangsu Province (no. BE2011368), Fundamental Research Funds for Central Universities (no. Y0201100249), the CAS/SAFEA International Partnership Program for Creative Research Teams, Yantai Doublehundred High-end Talent Plan (XY-003-02), and the Project of a Special Fund for Public Welfare Industrial (Agriculture) Research of China (no. 200903001-5).

\section{References}

[1] L. Pan, M. R. Sinden, A. H. Kennedy et al., "Bioactive constituents of Helianthus tuberosus (Jerusalem artichoke)," Phytochemistry Letters, vol. 2, no. 1, pp. 15-18, 2009.

[2] H. Baba, Y. Yaoita, and M. Kikuchi, "Sesquiterpenoids from the leaves of Helianthus tuberosus L.," Journal of Tohoku Pharmaceutical University, vol. 52, pp. 21-25, 2005.

[3] M. Talipova, "Lipids of Helianthus tuberosus," Chemistry of Natural Compounds, vol. 37, no. 3, pp. 213-215, 2001.

[4] R. R. Stange Jr., S. L. Midland, G. J. Holmes, J. J. Sims, and R. T. Mayer, "Constituents from the periderm and outer cortex of Ipomoea batatas with antifungal activity against Rhizopus stolonifer," Postharvest Biology and Technology, vol. 23, no. 2, pp. 85-92, 2001.

[5] X. Yuax, M. Gao, K. Wang, H. Xiao, C. Tan, and Y. Du, "Analysis of chlorogenic acids in Helianthus tuberosus Linn leaves using high performance liquid chromatography-mass spectrometry," 
Chinese Journal of Chromatography, vol. 26, no. 3, pp. 335-338, 2008.

[6] X. Yuan, M. Gao, H. Xiao, C. Tan, and Y. Du, "Free radical scavenging activities and bioactive substances of Jerusalem artichoke (Helianthus tuberosus L.) leaves," Food Chemistry, vol. 133, no. 1, pp. 10-14, 2012.

[7] M. S. Ahmed, F. S. El-Sakhawy, S. N. Soliman, and D. M. R. Abou-Hussein, "Phytochemical and biological study of Helianthus tuberosus L.," Egyptian Journal of Biomedical Science, vol. 18, pp. 134-147, 2005.

[8] U. Jin, J. Lee, S. Kang et al., "A phenolic compound, 5caffeoylquinic acid (chlorogenic acid), is a new type and strong matrix metalloproteinase- 9 inhibitor: isolation and identification from methanol extract of Euonymus alatus," Life Sciences, vol. 77, no. 22, pp. 2760-2769, 2005.

[9] P. Mattila and J. Hellström, "Phenolic acids in potatoes, vegetables, and some of their products," Journal of Food Composition and Analysis, vol. 20, no. 3-4, pp. 152-160, 2007.

[10] M. Friedman, "Chemistry, biochemistry, and dietary role of potato polyphenols. A review," Journal of Agricultural and Food Chemistry, vol. 45, no. 5, pp. 1523-1540, 1997.

[11] G. C. Percival, M. S. Karim, and G. R. Dixon, "Pathogen resistance in aerial tubers of potato cultivars," Plant Pathology, vol. 48, no. 6, pp. 768-776, 1999.

[12] S. L. Sinden, L. L. Sanford, W. W. Cantelo, and K. L. Deahl, "Bioassays of segregating plants-a strategy for studying chemical defenses," Journal of Chemical Ecology, vol. 14, no. 10, pp. 1941-1950, 1988.

[13] A. Wen, P. Delaquis, K. Stanich, and P. Toivonen, "Antilisterial activity of selected phenolic acids," Food Microbiology, vol. 20, no. 3, pp. 305-311, 2003.

[14] F. Tesio, L. A. Weston, and A. Ferrero, "Allelochemicals identified from Jerusalem artichoke (Helianthus tuberosus L.) residues and their potential inhibitory activity in the field and laboratory," Scientia Horticulturae, vol. 129, no. 3, pp. 361-368, 2011.

[15] F. Ferreres, R. Figueiredo, S. Bettencourt et al., "Identification of phenolic compounds in isolated vacuoles of the medicinal plant Catharanthus roseus and their interaction with vacuolar class III peroxidase: an $\mathrm{H}_{2} \mathrm{O}_{2}$ affair?" Journal of Experimental Botany, vol. 62, no. 8, pp. 2841-2854, 2011.

[16] M. Tchoné, G. Bärwald, G. Annemüller, and L. G. Fleischer, "Separation and identification of phenolic compounds in Jerusalem artichoke (Helianthus tuberosus L.)," Sciences des Aliments, vol. 26, no. 5, pp. 394-408, 2006.

[17] C. Alcalde-Eon, G. Saavedra, S. De Pascual-Teresa, and J. C. Rivas-Gonzalo, "Identification of anthocyanins of pinta boca (Solanum stenotomum) tubers," Food Chemistry, vol. 86, no. 3, pp. 441-448, 2004.

[18] Z. Charrouf, M. Hilali, O. Jauregui, M. Soufiaoui, and D. Guillaume, "Separation and characterization of phenolic compounds in argan fruit pulp using liquid chromatographynegative electrospray ionization tandem mass spectroscopy," Food Chemistry, vol. 100, no. 4, pp. 1398-1401, 2007.

[19] Z. Fang, M. Zhang, and L. Wang, "HPLC-DAD-ESIMS analysis of phenolic compounds in bayberries (Myrica rubra Sieb. et Zucc.)," Food Chemistry, vol. 100, no. 2, pp. 845-852, 2007.

[20] L. Longo and G. Vasapollo, "Extraction and identification of anthocyanins from Smilax aspera L. berries," Food Chemistry, vol. 94, no. 2, pp. 226-231, 2006.

[21] R. Pedreschi and L. Cisneros-Zevallos, "Phenolic profiles of Andean purple corn (Zea mays L.)," Food Chemistry, vol. 100, no. 3, pp. 956-963, 2007.
[22] A. P. Rauter, A. Martins, C. Borges et al., "Liquid chromatography-diode array detection-electrospray ionisation mass spectrometry/nuclear magnetic resonance analyses of the antihyperglycemic flavonoid extract of Genista tenera: structure elucidation of a flavonoid-C-glycoside," Journal of Chromatography A, vol. 1089, no. 1-2, pp. 59-64, 2005.

[23] N. P. Seeram, R. Lee, H. S. Scheuller, and D. Heber, "Identification of phenolic compounds in strawberries by liquid chromatography electrospray ionization mass spectroscopy," Food Chemistry, vol. 97, no. 1, pp. 1-11, 2006.

[24] Y. Zu, C. Li, Y. Fu, and C. Zhao, "Simultaneous determination of catechin, rutin, quercetin kaempferol and isorhamnetin in the extract of sea buckthorn (Hippophae rhamnoides L.) leaves by RP-HPLC with DAD," Journal of Pharmaceutical and Biomedical Analysis, vol. 41, no. 3, pp. 714-719, 2006.

[25] P. Hu, Q. Liang, G. Luo, Z. Zhao, and Z. Jiang, "Multicomponent HPLC fingerprinting of Radix Salviae Miltiorrhizae and its LC-MS-MS identification," Chemical and Pharmaceutical Bulletin, vol. 53, no. 6, pp. 677-683, 2005.

[26] X. LONG, J. CHI, L. LIU, Q. LI, and Z. LIU, "Effect of seawater stress on $\mathrm{p}$ hysiological and biochemical responses offive Jerusalem a rtichoke ecotypes," Pedosphere, vol. 19, no. 2, pp. 208-216, 2009.

[27] X. H. Long, J. Tian, Q. W. Zhong, Z. R. Huang, L. Li, and Z. P. Liu, "Study the cultivars comparison of Helianthus Tuberosus L. and planting technology of low cost and high quality in noninfield of Qinghai and Xinjiang Provinces," Chinese Agricultural Science Bulletin, vol. 26, no. 13, pp. 354-358, 2010.

[28] Z. Huang, X. Long, L. Wang et al., "Growth, photosynthesis and $\mathrm{H}^{+}$-ATPase activity in two Jerusalem artichoke varieties under NaCl-induced stress," Process Biochemistry, vol. 47, no. 4, pp. 591-596, 2012.

[29] K. Tawaha, F. Q. Alali, M. Gharaibeh, M. Mohammad, and T. El-Elimat, "Antioxidant activity and total phenolic content of selected Jordanian plant species," Food Chemistry, vol. 104, no. 4, pp. 1372-1378, 2007.

[30] K. Zhou and L. Yu, "Total phenolic contents and antioxidant properties of commonly consumed vegetables grown in Colorado," LWT-Food Science and Technology, vol. 39, no. 10, pp. 1155-1162, 2006.

[31] R. Jaiswal, S. Deshpande, and N. Kuhnert, "Profling the chlorogenic acids of Rudbeckia hirta, Helianthus tuberosus, Carlina acaulis and symphyotrichum novae-angliae leavesby LC-MS ${ }^{n}$," Phytochemical Analysis, vol. 22, no. 5, pp. 432-441, 2011.

[32] L. Bravo, L. Goya, and E. Lecumberri, "LC/MS characterization of phenolic constituents of mate (Ilex paraguariensis, St. Hil.) and its antioxidant activity compared to commonly consumed beverages," Food Research International, vol. 40, no. 3, pp. 393405, 2007.

[33] X. Wang, W. Sun, H. Sun et al., "Analysis of the constituents in the rat plasma after oral administration of Yin Chen Hao Tang by UPLC/Q-TOF-MS/MS," Journal of Pharmaceutical and Biomedical Analysis, vol. 46, no. 3, pp. 477-490, 2008.

[34] M. Križman, D. Baričevič, and M. Prošek, "Determination of phenolic compounds in fennel by HPLC and HPLCMS using a monolithic reversed-phase column," Journal of Pharmaceutical and Biomedical Analysis, vol. 43, pp. 481-485, 2007.

[35] A. Tolonen, T. Joustamo, S. Mattlla, T. Kämäräinen, and J. Jalonen, "Identification of isomeric dicaffeoylquinic acids from Eleutheracoccus senticosus using HPLC-ESI/TOF/MS and HNMR methods," Phytochemical Analysis, vol. 13, no. 6, pp. 316$328,2002$. 
[36] M. N. Clifford, W. Zheng, and N. Kuhnert, "Profiling the chlorogenic acids of aster by HPLC-MSn," Phytochemical Analysis, vol. 17, no. 6, pp. 384-393, 2006.

[37] M. N. Clifford, J. Kirkpatrick, N. Kuhnert, H. Roozendaal, and P. R. Salgado, "LC-MS ${ }^{n}$ analysis of the cis isomers of chlorogenic acids," Food Chemistry, vol. 106, no. 1, pp. 379-385, 2008.

[38] R. Jaiswal, J. Kiprotich, and N. Kuhnert, "Determination of the hydroxycinnamate profile of 12 members of the Asteraceae family," Phytochemistry, vol. 72, no. 8, pp. 781-790, 2011.

[39] J. Pan and Y. Cheng, "Identification and analysis of absorbed and metabolic components in rat plasma after oral administration of "Shuangdan" granule by HPLC-DAD-ESI-MS/MS," Journal of Pharmaceutical and Biomedical Analysis, vol. 42, no. 5, pp. 565-572, 2006.

[40] C. Tian, X. Xu, L. Liao, J. Zhang, J. Liu, and S. Zhou, "Separation and identification of chlorogenic acid and related impurities by high performance liquid chromatography-tandem mass spectrometry," Chinese Journal of Chromatography, vol. 25, no. 4, pp. 496-500, 2007.

[41] L. Zhang, C. Fan, X. Zhang, Z. Yin, and W. Ye, "A new steroidal glycoside from Lygodium japonicum," Journal of China Pharmaceutical University, vol. 37, no. 6, pp. 491-493, 2006.

[42] K. Ablajan, Z. Abliz, X. Shang, J. He, R. Zhang, and J. Shi, "Structural characterization of flavonol 3,7-di-O-glycosides and determination of the glycosylation position by using negative ion electrospray ionization tandem mass spectrometry," Journal of Mass Spectrometry, vol. 41, no. 3, pp. 352-360, 2006.

[43] P. Kachlicki, J. Einhorn, D. Muth, L. Kerhoas, and M. Stobiecki, "Evaluation of glycosylation and malonylation patterns in flavonoid glycosides during LC/MS/MS metabolite profiling," Journal of Mass Spectrometry, vol. 43, no. 5, pp. 572-586, 2008.

[44] F. Sánchez-Rabaneda, O. Jáuregui, I. Casals, C. AndrésLacueva, M. Izquierdo-Pulido, and R. M. Lamuela-Raventós, "Liquid chromatographic/electrospray ionization tandem mass spectrometric study of the phenolic composition of cocoa (Theobroma cacao)," Journal of Mass Spectrometry, vol. 38, no. 1, pp. 35-42, 2003.

[45] Z. He and W. Xia, "Analysis of phenolic compounds in Chinese olive (Canarium album L.) fruit by RPHPLC-DAD-ESI-MS," Food Chemistry, vol. 105, no. 3, pp. 1307-1311, 2007.

[46] A. Schieber, P. Keller, and R. Carle, "Determination of phenolic acids and flavonoids of apple and pear by high-performance liquid chromatography," Journal of Chromatography A, vol. 910, no. 2, pp. 265-273, 2001.

[47] G. G. Duthie, S. J. Duthie, and J. A. M. Kyle, "Plant polyphenols in cancer and heart disease: implications as nutritional antioxidants," Nutrition Research Reviews, vol. 13, no. 1, pp. 79-106, 2000.

[48] J. H. Doughari, I. S. Human, S. Bennade, and P. A. Ndakidemi, "Phytochemicals as chemotherapeutic agents and antioxidants: possible solution to the control of antibiotic resistant verocytotoxin producing bacteria," Journal of Medicinal Plants Research, vol. 3, no. 11, pp. 839-848, 2009.

[49] C. H. Lu, X. G. Liu, J. Xu et al., "Enhanced exudation of DIM$\mathrm{BOA}$ and $\mathrm{MBOA}$ by wheat seedlings alone and in proximity to wild oat (Avena fatua) and flixweed (Descurainia sophia)," Weed Science, vol. 60, no. 3, pp. 360-365, 2012.

[50] K. Schrader, A. Kiehne, U. H. Engelhardt, and H. G. Maier, "Determination of chlorogenic acids with lactones in roasted coffee," Journal of the Science of Food and Agriculture, vol. 71, no. 3, pp. 392-398, 1996.
[51] C. Manach, A. Scalbert, C. Morand, C. Rémésy, and L. Jiménez, "Polyphenols: food sources and bioavailability", The American Journal of Clinical Nutrition, vol. 79, no. 5, pp. 727-747, 2004.

[52] X. Duan, G. Wu, and Y. Jiang, "Evaluation of the antioxidant properties of litchi fruit phenolics in relation to pericarp browning prevention," Molecules, vol. 12, no. 4, pp. 759-771, 2007. 

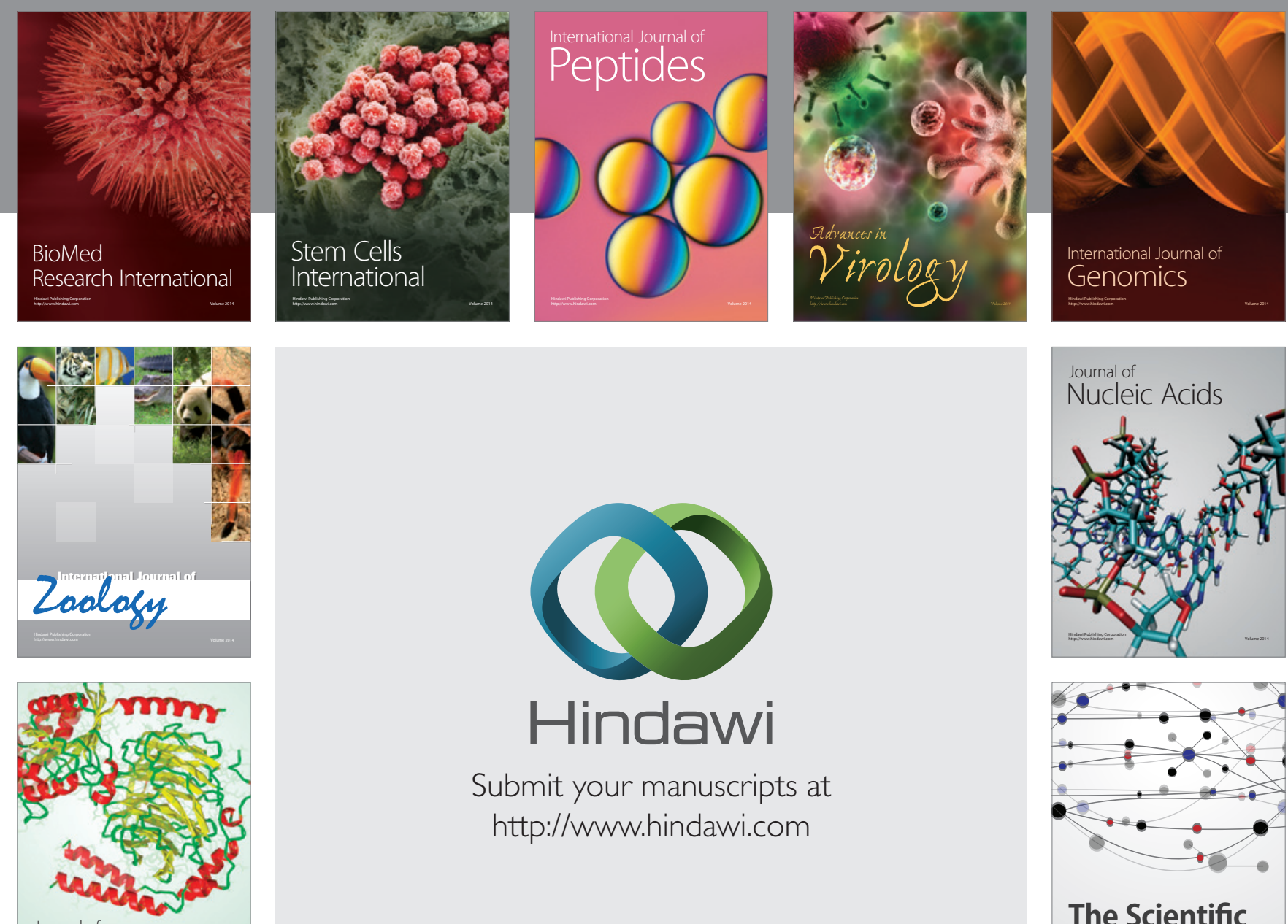

Submit your manuscripts at

http://www.hindawi.com

Journal of
Signal Transduction
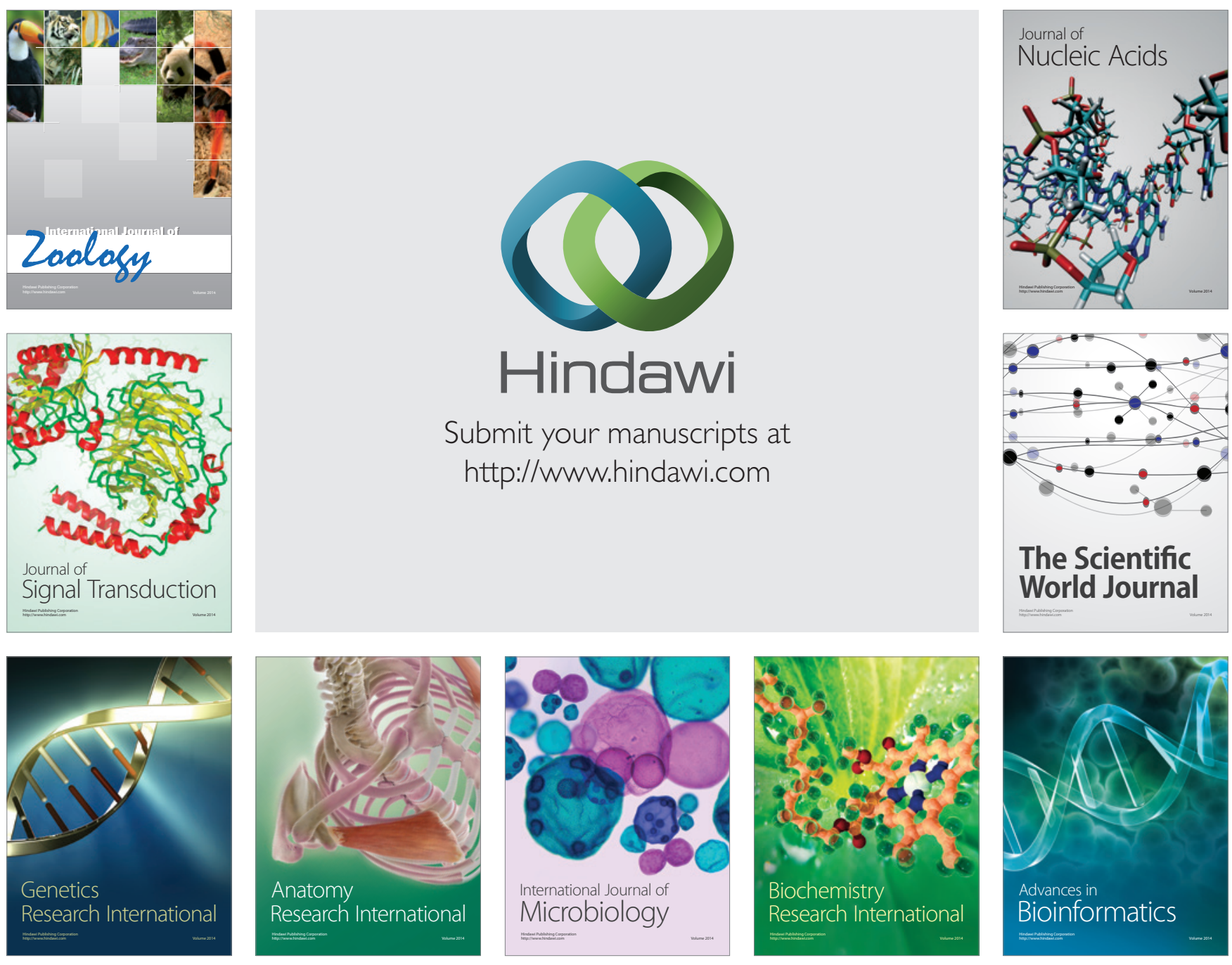

The Scientific World Journal
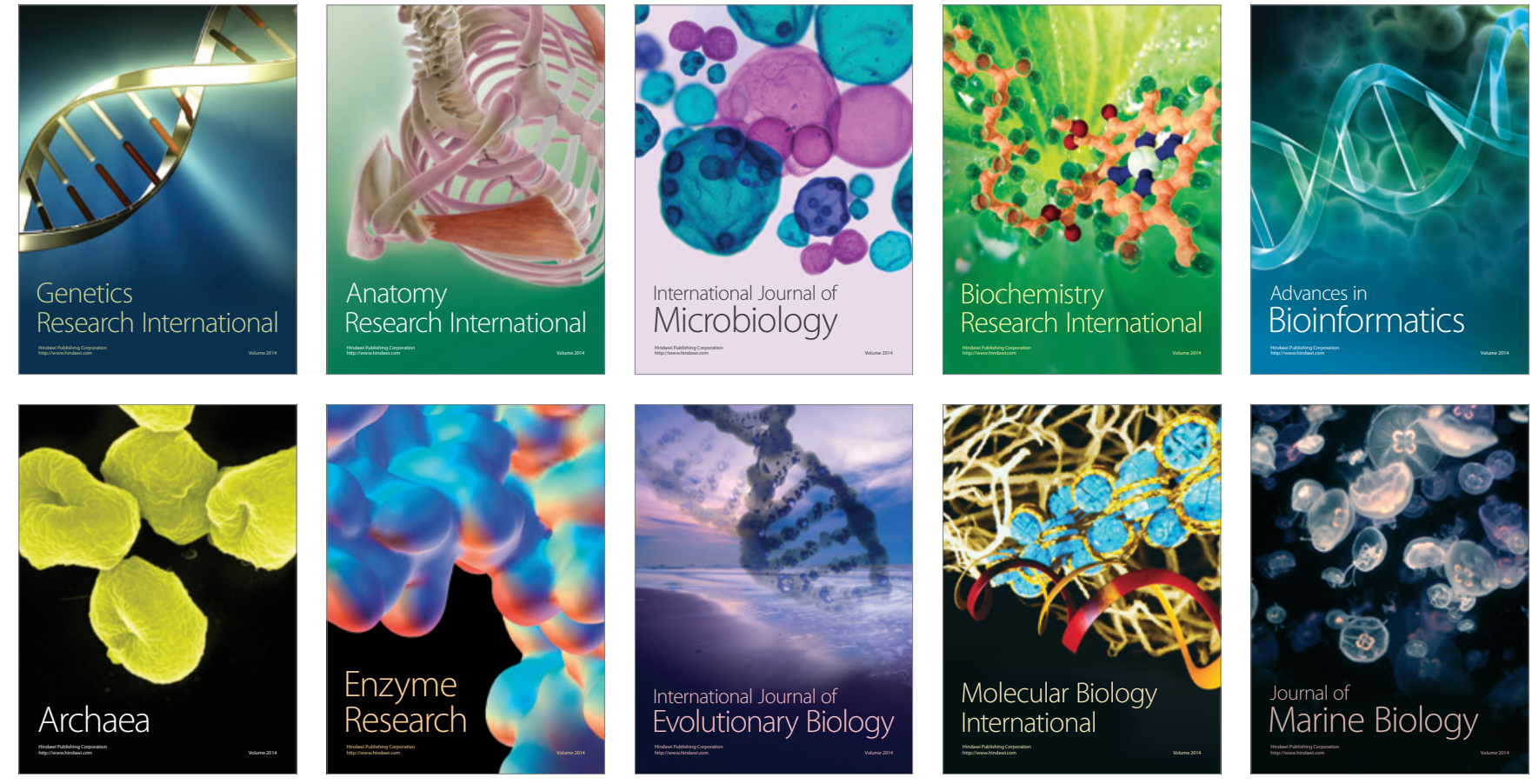